\title{
ARHGAP24 regulates cell ability and apoptosis of colorectal cancer cells via the regulation of P53
}

\author{
SUILIANG ZHANG ${ }^{1 *}$, LIANG SUI $^{2 *}$, JUHUA ZHUANG $^{3}$, SAIFEI HE $^{3}$, YANAN SONG ${ }^{3}$, YING YE $^{3}$ and WEI XIA ${ }^{3}$ \\ ${ }^{1}$ Department of Oncology and Interventional Therapy, The Seventh People's Hospital of Shanghai University \\ of Traditional Chinese Medicine, Shanghai 200137; ${ }^{2}$ Department of Emergency, Ruijin Hospital, \\ Medical School of Shanghai Jiaotong University, Shanghai 200025; ${ }^{3}$ Department of Nuclear Medicine, \\ The Seventh People's Hospital of Shanghai University of Traditional Chinese Medicine, Shanghai 200137, P.R. China
}

Received September 29, 2017; Accepted June 11, 2018

DOI: $10.3892 / \mathrm{ol} .2018 .9075$

\begin{abstract}
Colorectal cancer is a human malignancy ranked the third highest of the global incidence of malignant tumors. Rho GTPase-activating proteins (RHOGAPs) were identified functional in several processes of tumors. In the present study, through reverse transcription-quantitative PCR (RT-qPCR) and western blot analysis, expression of Rho GTPase-activating protein 24 (ARHGAP24) and p53 was measured in colorectal cancer tissues, which was lower than that in adjacent normal tissues, revealing that ARHGAP24 may be implicated in the progress of colorectal cancer and in vitro, overexpression of ARHGAP24 in LoVo and HCT116 cells inhibited the cell ability and enhanced cell apoptosis, and accompanied with high protein expression of p53, p21 and Bax. Further, addition of p53 inhibitor PFT- $\alpha$ had an antagonistic effect on cell proliferation and apoptosis of LoVo and HCT116 cells induced by ARHGAP24 overexpression. In addition, the expression of p21 and Bax was positively correlated with p53 expression. All of the above data demonstrated that ARHGAP24 was likely to be a tumor suppressor in colorectal cancer and may function closely related to p53, p21 and Bax. We inferred that ARHGAP24 may be a novel target for in-depth study of colorectal cancer.
\end{abstract}

\section{Introduction}

Colorectal cancer is a malignancy that occurs in the gut, and the incidence is second only to the stomach and esophageal

Correspondence to: Dr Ying Ye or Dr Wei Xia, Department of Nuclear Medicine, The Seventh People's Hospital of Shanghai University of Traditional Chinese Medicine, 358 Datong Road, Pudong, Shanghai 200137, P.R. China

E-mail: yy49453324@163.com

E-mail: awingxia_d@126.com

*Contributed equally

Key words: ARHGAP24, colorectal cancer, p53, p21, Bax, cell ability, apoptosis cancer, and it is a common malignancy in the gastrointestinal tract. In addition to genetics, behavioral and environmental causes are also closely related to its incidence (1-3). It is one of the main causes of cancer-related deaths worldwide, especially in Western countries (4-7). Due to high recurrence rates and metastases, the mortality rate caused by colorectal cancer remains high despite the treatment of colorectal cancer has improved with development of technology $(8,9)$. Thus, new promising targets for therapy of colorectal cancer are urgently needed.

Rho GTPase-activating protein 24 (ARHGAP24), belongs to Rho GTPase-activating proteins (RHOGAPs) family, is a protein containing 748 amino acids involved in cell cycle, apoptosis and invasion and other cell processes. As for tumor research, several RHOGAP proteins were found implicated in human tumors. For example, it is revealed that upregulation of ARHGAP10 had an inhibitory effect on tumorigenicity of ovarian cancer cells (10), and ARHGAP10 also acted as a tumor suppressor in lung cancer (11). Besides ARHGAP10, ARHGAP35 and ARHGAP8 were also reported as candidate tumor suppressors functionig in colorectal cancer $(12,13)$. A study has related the function of ARHGAP24 to the cell apoptosis and invasion of renal cell carcinoma (14). However, there is little research on the function of ARHGAP24 in colorectal cancer, so here we aim to investigated the functions of ARHGAP24 in colorectal cancer.

Additionally, the studies of tumor usually focus on cell apoptosis and the cell cycle process, which are associated with p53. p53, mutated in $\sim 50 \%$ of all malignant neoplasm, is a well-known tumor suppressor gene of human cancer. It is reported that the mutation of p53 can cause alteration of growth arrest and deficient apoptosis (15).

In the present study, low expression of ARHGAP24 and p53 was noted in tumor tissues of colorectal cancer patients revealed that ARHGAP24 may function in colorectal cancer via p53. In vitro, upregulation of ARHGAP24 in colorectal cancer cell lines inhibited the cell ability, in contrast, apoptotic cells were significantly increased accompanied with high expression of apoptosis-related proteins p53, p21 and Bax. The addition of p53 inhibitor PFT- $\alpha$ antagonized the induction of ARHGAP24 on cell ability of colorectal cancer cells. The protein level of p21 and Bax were correspondingly declined by 
PFT- $\alpha$. Based on the above, we conjectured that ARHGAP24 may affect colorectal cancer via the regulation of p53, p21 and Bax. Targeting ARHGAP24 may provide a potential promising direction for research and therapy of colorectal cancer.

\section{Materials and methods}

Colorectal cancer and adjacent normal tissues. With written informed consent, thirty colorectal cancer and paired adjacent normal tissues were collected from the colorectal cancer patients treated in The Seventh People's Hospital of Shanghai University of Traditional Chinese Medicine (Shanghai, China) who volunteered to participate in the study. Another four pairs of colorectal cancer and adjacent normal tissues were also collected. Before used, the tissue samples were stored in liquid nitrogen. The study was approved by the Ethics Committee of The Seventh People's Hospital of Shanghai University of Traditional Chinese Medicine.

Cell culture. LoVo and HCT116, two human colorectal cancer cell lines, were obtained from Cell Bank of Chinese Academy of Science (Shanghai, China). Generally, in a $37^{\circ} \mathrm{C}, 5 \% \mathrm{CO}_{2}$ incubator (Thermo Fisher Scientific, Inc., Waltham, MA, USA), LoVo and HCT116 cells were respectively cultured in 1640 medium (HyClone; GE Healthcare Life Sciences, Logan, UT, USA) and DMEM high glucose medium (HyClone; GE Healthcare Life Sciences) both containing 10\% fetal bovine serum (Gibco; Thermo Fisher Scientific, Inc.) and 1\% antibiotic (admixture of penicillin and streptomycin; Beijing Solarbio Science \& Technology Co., Ltd., Beijing, China). The medium was replaced according to the cell growth state during incubation.

The construction of lentivirus. First, vector pLVX-Puro was constructed according to the experimental requirements. The 2247 bp length coding DNA sequence (CDS) region of ARHGAP24 containing cleavage sites of EcoR I and BamH I was then synthesized by Genewiz Company (Shanghai, China), which was then inserted into EcoR I/BamH I sites of pLVX-Puro and confirmed by DNA sequencing (Majorbio, Shanghai, China). Next, through Lipofectamine ${ }^{\text {TM }} 2000$ (Invitrogen; Thermo Fisher Scientific, Inc.), lentivirus core plasmid pLVX-Puro-ARHGAP24 (Clontech Laboratories, Inc., Mountainview, CA, USA) and two packaging plasmids psPAX2 and pMD2G (Addgene, Inc., Cambridge, MA, USA) were co-tranfected into $293 \mathrm{~T}$ cells. After $48 \mathrm{~h}$ the virus particles in the supernatant were collected by ultracentrifugation (16).

Experimental group. LoVo cells were divided into three groups to infect with ARHGAP24 lentivirus. Grouped as follows, LoVo cells were counted and infected with lentivirus of ARHGAP24 overexpression (oeARHGAP24)/empty vectors (Vector). Wild-type LoVo cells only cultured with medium were used as control. On the other hand, HCT116 cells were also grouped following the above. Subsequently, RT-qPCR and western blot analysis were carried out to detect the overexpression efficiency of ARHGAP24. The assays of the cell ability and apoptosis were also performed.

In addition, to investigate the effect of p53 on oeARHGAP24-induced LoVo cells, cells were grouped as follows.
Vector, LoVo cells were infected with empty vectors; oeARHGAP24, LoVo cells were infected with ARHGAP24 overexpression lentivirus; oeARHGAP24+PFT- $\alpha$, LoVo cells were treated with oeARHGAP24 and PFT- $\alpha$ (20 $\mu \mathrm{g} / \mathrm{l})$. Next, cell ability assay and western blot analysis were carried out.

Immunohistochemical detection. The embedded and fixed tissues were cut into 4-7 $\mu \mathrm{m}$ slices. The slices were grilled in a $65^{\circ} \mathrm{C}$ constant temperature oven for $30 \mathrm{~min}$, soaked in xylene I (Sinopharm Chemical Reagent Co., Ltd., Shanghai, China) for $15 \mathrm{~min}$, and then soaked in xylene II for $15 \mathrm{~min}$. The dewaxed-slices were soaked with gradient concentrations of ethanol $(100,95,85$, and $75 \%)$ and each gradient for $5 \mathrm{~min}$, followed by $10 \mathrm{~min}$ flushing of tap water. After antigen retrieval with $0.01 \mathrm{M}$ citrate buffer solution for $15 \mathrm{~min}$, the slices were incubated in wet-box with $0.3 \% \mathrm{H}_{2} \mathrm{O}_{2}$ for $10 \mathrm{~min}$. Later, following incubation with rabbit polyclonal ARHGAP24 antibody (1:200; cat. no. Ab203874; Abcam, Cambridge, $\mathrm{UK}$ ) in wet-box at room temperature for $1 \mathrm{~h}$, the slices were incubated with secondary goat anti-rabbit (HRP) IgG antibody (1:2000; cat. no. ab6721; Abcam) at room for 20-30 min. Subsequently, the slices were treated with DAB, 3 min staining of hematoxylin (714094; Baso Diagnostic, Inc., Wuhan, China), $1 \%$ hydrochloric acid for alcohol differentiation. After 10 min flushing of tap water, the slices were grilled, made transparent and closed. Finally, the images were observed under microscopy (CX41; Olympus Corporation, Tokyo, Japan) and analyzed by IMS image analysis system (Shanghai Jierdun Biotech Co., Ltd., Shanghai, China).

Cell Counting Kit-8 (CCK-8) assay. Logarithmic growth phase cells after digested by $0.25 \%$ trypsin were seeded in 96-well plates with $100 \mu \mathrm{l}$ of cell suspension $\left(3 \times 10^{4}\right.$ cells $\left./ \mathrm{ml}\right)$ added to each well, then cultured overnight. Cells were treated according to the above protocol, and then $100 \mu \mathrm{l}$ mixtures of 10\% CCK-8 (Sigma-Aldrich; Merck KGaA, Darmstadt, Germany Inc.) solution in serum-free medium were added to each well with $1 \mathrm{~h}$ of incubation. After that, a microplate reader (Perlong, Beijing, China) was applied to measure the absorbance value (OD) of each well at $450 \mathrm{~nm}$.

Reverse transcription-quantitative PCR (RT-qPCR) assay. Total RNA was extracted from LoVo and HCT116 cells by using TRIzol reagent (Invitrogen; Thermo Fisher Scientific, Inc.), and then confirmed by $1 \%$ agarose gel electrophoresis after quantification. Subsequently, through reverse transcriptase kit (Fermentas; Thermo Fisher Scientific, Inc.), cDNA was synthesized from the isolated RNA. After that, following the procedure of RT-qPCR reactions, the gene expression was analyzed by a machine of ABI Prism 7300 (ABI; Thermo Fisher Scientific, Inc.) using SYBR-Green PCR kit (Thermo Fisher Scientific, Inc.). The mRNA expression of ARHGAP24 was analyzed by application of $2^{-\Delta \Delta C q}$ method with GAPDH as an internal control (17). The primers were as follows: ARHGAP24, 5'-AACTCCTGTCG CTCTTCTACC-3' and 5'-GCTGTTGCCCACAAATGTCT C-3'; p53, 5'-CCACCATCCACTACAAC TAC-3' and 5'-AAA CACGCACCTCAA AGC -3'; GAPDH, 5'-CACCCACTCCT CCACCTTTG-3' and 5'-CCACCACCCTGTTGCTGTAG-3'. The RT-qPCR was conducted as the following procedure: 
cycle $1,95^{\circ} \mathrm{C}$ for $10 \mathrm{~min}$; cycle 2 with 40 repeated cycles of $95^{\circ} \mathrm{C}$ for $15 \mathrm{sec}, 60^{\circ} \mathrm{C}$ for $45 \mathrm{sec}$, and then $95^{\circ} \mathrm{C}$ for $15 \mathrm{sec}, 60^{\circ} \mathrm{C}$ for $1 \mathrm{~min}$ for one cycle; $95^{\circ} \mathrm{C}$ for $15 \mathrm{sec}, 60^{\circ} \mathrm{C}$ for $15 \mathrm{sec}$ for one cycle $(18,19)$.

Western blot analysis. Treated cells were washed with cold phosphate-buffered saline (PBS) twice and then lysed in a RIPA buffer (Solarbio Science \& Technology Co., Ltd.) which contained protease and phosphatase inhibitors for $\sim 30 \mathrm{~min}$ on ice. A pre-cooled centrifuge was applied to centrifuge the lysates and the protein in the supernatant was obtained. Subsequently, the isolated protein was calculated by BCA method (Thermo Fisher Scientific, Inc.). Every $15 \mu 1$ extracted protein used for one sample were subjected to 15 and $10 \%$ SDS-PAGE (JRDUN Biotechnology Co., Ltd, Shanghai, China) and semi-dry transferred onto polyvinylidene fluoride (PVDF) membranes (EMD Millipore, Billerica, MA, USA). Following blocking with 5\% skim milk (BD Biosciences, San Jose, CA, USA) in PBST, the membranes were incubated with primary antibodies, ARHGAP24 (cat. no. Ab203874; 1:200; Abcam, Cambridge, UK), Bax (cat. no. Sc-493; 1:300, Santa Cruz Biotechnology, Inc., Dallas, TX, USA), p21 (1:1,000; cat. no. 2947), GAPDH (1:2,000; cat. no. 5174) and p53 (cat. no. 2524; 1:1,000) all from Cell Signaling Technology, Inc., (Danvers, MA, USA) at $4^{\circ} \mathrm{C}$ overnight with gentle shaking (at room for $2 \mathrm{~h}$ ), followed by washing with PBST 6 times. After incubation of secondary goat anti-rabbit (HRP) IgG antibody (1:2,000; cat. no. ab6721; Abcam) for $2 \mathrm{~h}$ at at $37^{\circ} \mathrm{C}$ in the dark, the membranes were washed with PBST 6 times again. Finally, using a chemiluminescence detection reagent (Millipore), the blots were visualized by an instrument of ECL chemiluminescence (Tanon Science and Technology Co., Ltd., Shanghai, China).

Cell cycle detection. Colorectal cancer cells of LoVo and HCT116 were infected with oeARHGAP24 lentivirus. After $48 \mathrm{~h}$ of infection, the cells were centrifuged at 1,000 $\mathrm{x}$ g for $5 \mathrm{~min}$, and then resuspended with $300 \mu \mathrm{l}$ PBS containing $10 \%$ fetal bovine serum (Gibco; Thermo Fisher Scientific, Inc.). Then $700 \mu \mathrm{l}$ of absolute ethanol was added for fixing at $-20^{\circ} \mathrm{C}$ overnight. The next day, the fixed-cells were centrifuged at 3,000 x g for $30 \mathrm{sec}$ and washed twice with $1 \mathrm{ml}$ pre-cooled PBS. The cell pellets were resuspended with $100 \mu 1 \mathrm{of} 1 \mathrm{mg} / \mathrm{ml}$ RNase A solution and incubated at $37^{\circ} \mathrm{C}$. Subsequently, the cells were stained with a $400 \mu \mathrm{l}$ of $50 \mu \mathrm{g} / \mathrm{ml}$ propidium iodide (PI) solutions (Shanghai Beiyi Bioequip Information Co., Ltd.) in the dark for $10 \mathrm{~min}$. Finally, the numbers of LoVo and HCT116 cells in each phase of the cell cycle were analyzed by BD flow cytometry.

Flow cytometry (FCM) analysis. Apoptotic cells were analyzed by FCM (BD Biosciences) using Annexin V-fluorescein isothiocyanate (FITC)/propidium iodide (PI) double staining (Shanghai Beiyi Bioequip Information Co., Ltd.), as follows. After washing, digestio, resuspension and counting, $\sim 5 \times 10^{5}-1 \times 10^{6}$ of the treated cells was obtained after centrifugation for $5 \mathrm{~min}$ at 1,000 x g. Resuspended cells were precipitated with $195 \mu \mathrm{l}$ of Annexin V-FITC binding buffer, $5 \mu \mathrm{l}$ Annexin V-FITC was added to incubate the cells at $4^{\circ} \mathrm{C}$ for $15 \mathrm{~min}$ in the dark. Under the same conditions, the cells were then incubated with $5 \mu \mathrm{l}$ PI for $5 \mathrm{~min}$, while a tube without Annexin V-FITC and PI was a control. Finally, cell apoptosis rates were determined by BD flow cytometry.

Statistical analysis. Software of GraphPad prism 7.0 (GraphPad Software, Inc., La Jolla, CA, USA) was used to express all the statistical analyses. The differences of each two groups were evaluated by Student's t-test, while three and more comparisons were presented by one way analysis of variance (ANOVA) followed by post hoc test (Least Significant Difference). All values are mean \pm standard deviation of at least three independent experiments. $\mathrm{P}<0.05$ was considered to indicate a statistically significant difference.

\section{Results}

ARHGAP24 and p53 low expression in tumor tissues of colorectal cancer patients. Thirty cancer tissues and paired adjacent normal tissues were collected from thirty colorectal cancer patients who volunteered to participate in this study. Further four pairs of these tissues were also obtained for protein detection. As shown in Fig. 1, immunohistochemical detections of tissues revealed that ARHGAP24 expression was significantly low in tumors of colorectal cancer patients (Fig. 1A). After RNA and proteins extraction, the mRNA and protein expression of ARHGAP24 and p53 was respectively detected by RT-qPCR and western blot analysis. We discovered that in colorectal cancer patients, the mRNA (Fig. 1B) as well as protein (Fig. 1C) level of ARHGAP24 and p53 was reduced obviously in cancer tissues compared to adjacent normal tissues. From this, ARHGAP24 and p53 were considered likely to be involved in colorectal cancer.

Overexpression of ARHGAP24 in LoVo and HCT116 cell lines. To further study the function of ARHGAP24 exerted in colorectal cancer, LoVo and HCT116 cell lines were infected with ARHGAP24 overexpressed lentivirus, respectively. After $48 \mathrm{~h}$ of infection, RT-qPCR and western blot analysis were carried to detect the overexpression efficiency of ARHGAP24. As presented in Fig. 2, both at the transcription and protein translation level, the expression of ARHGAP24 was significantly increased in LoVo (Fig. 2A and B) and HCT116 (Fig. 2C and D) cells. Therefore, ARHGAP24 overexpressed lentivirus was chosen to use in subsequent experiments.

Upregulation of ARHGAP24 inhibits the cell ability of colorectal cancer cells via p53, p21 and Bax expression. For exploring the effect of ARHGAP24 on cell ability of colorectal cancer, after infected with oeARHGAP24 lentivirus, counted LoVo and HCT116 cells were cultured with CCK-8 mixture for $0,24,48$, and $72 \mathrm{~h}$. Subsequently, the cell ability of LoVo and HCT116 cells were measured by a machine of microplate reader (Bio-Rad, Hercules, CA, USA). The results in Fig. 3 showed that ARHGAP24 overexpression inhibited the cell ability of LoVo cells in a time-dependent manner and had an obvious effect after $48 \mathrm{~h}$ of CCK-8 treatment (Fig. 3A). On the other hand, in HCT116 cells, overexpression of ARHGAP24 performed a similar effect (Fig. 3B). Further, ARHGAP24-infected LoVo cells were treated with PFT- $\alpha$, an inhibitor of p53, for $48 \mathrm{~h}$. We found that the addition of PFT- $\alpha$ significantly antagonized 
A
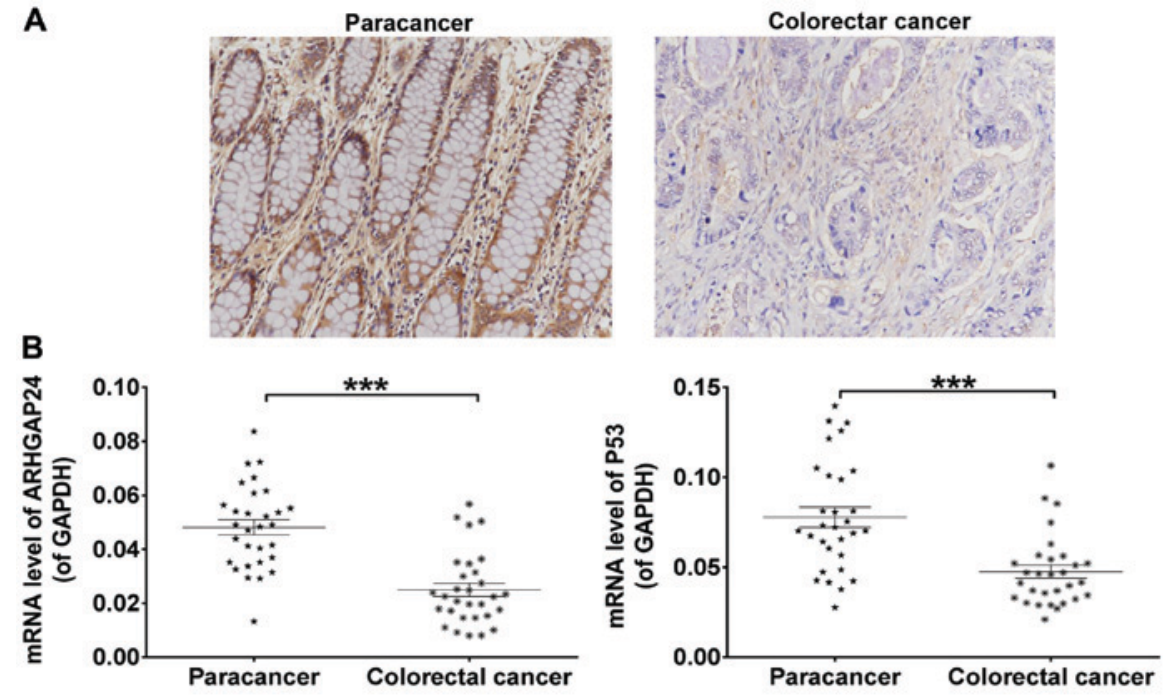

C

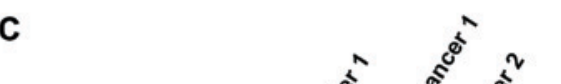

Colorectar cancer

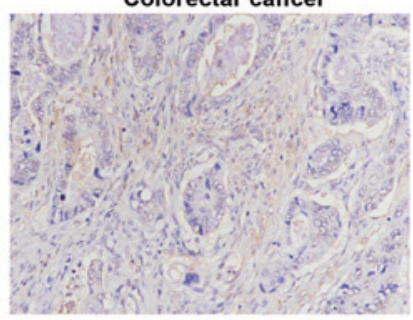

ARHGAP24
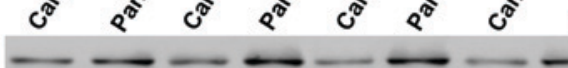

P53

GAPDH
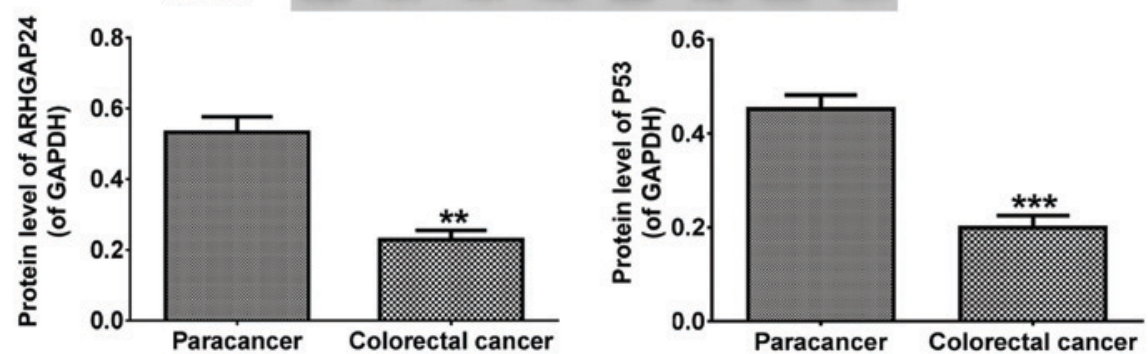

Figure 1. ARHGAP24 and 53 low expression in tumor tissues of colorectal cancer patients. Thirty samples and another four pairs of tumor and adjacent normal tissues were collected from colorectal cancer patients. (A) ARHGAP24 expression in these tissues was detected by immunohistochemisry. (B) RT-qPCR was carried to quantify the mRNA expression of ARHGAP24 and p53. (C) The protein levels of ARHGAP24 and p53 were quantified by western blot analysis. The data are mean \pm standard deviation (SD). ${ }^{* *} \mathrm{P}<0.01,{ }^{* * *} \mathrm{P}<0.001$ compared to paracancer. ARHGAP24, Rho GTPase-activating protein 24.

A

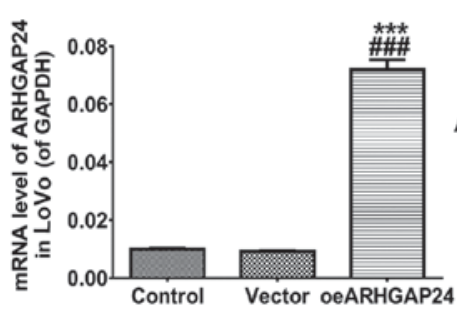

C

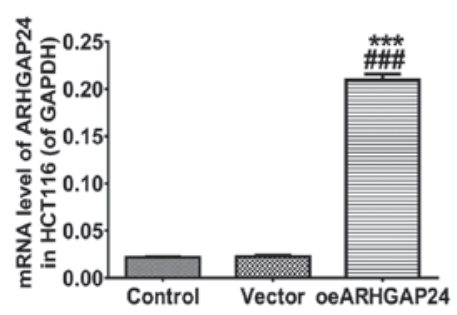

B
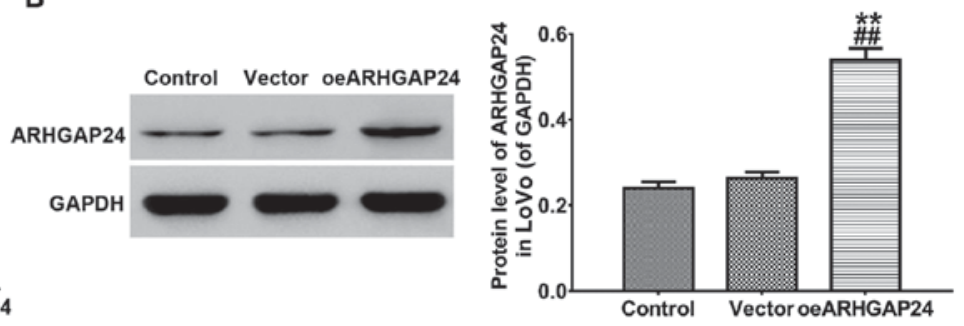

D
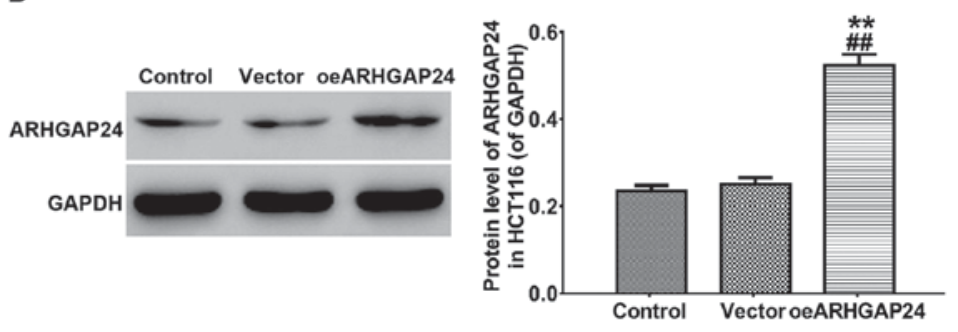

Figure 2. Overexpression of ARHGAP24 in LoVo and HCT116 cell lines. Cultured LoVo and HCT116 cells were both infected with ARHGAP24 overexpression lentivirus and after $48 \mathrm{~h}$ the cells were harvested. (A and B) ARHGAP24 mRNA and protein levels in LoVo cells were detected by RT-PCT. (C and D) Likewise, ARHGAP24 expression in HCT116 cells was also quantified. All data are the mean \pm standard deviation. ${ }^{* * *} \mathrm{P}<0.01,{ }^{* * * *} \mathrm{P}<0.001$ compared to control; ${ }^{\# \#} \mathrm{P}<0.01,{ }^{\# \# \#} \mathrm{P}<0.001$ compared to vector. ARHGAP24, Rho GTPase-activating protein 24. 
A

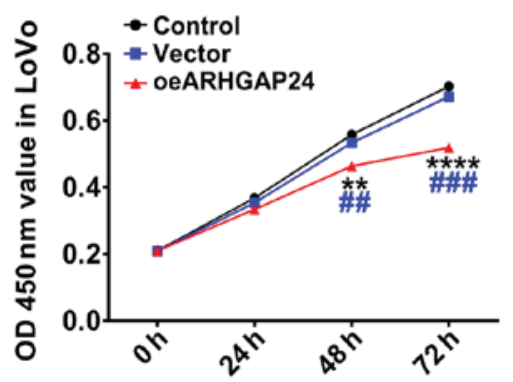

B

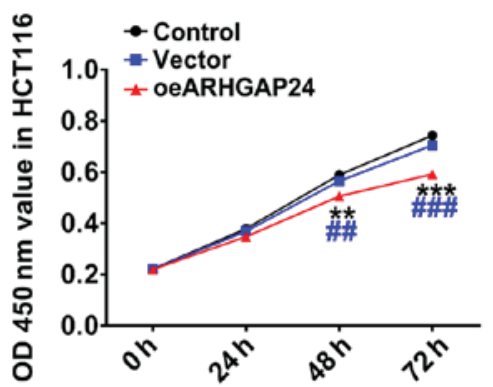

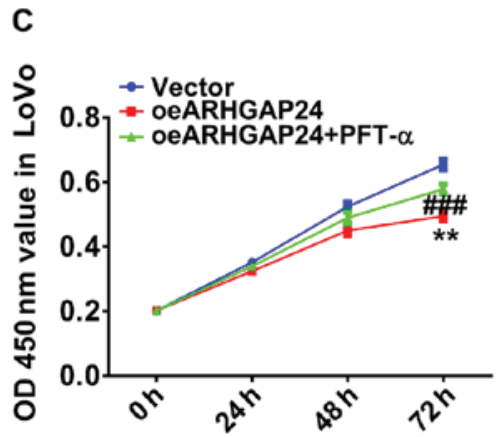

D
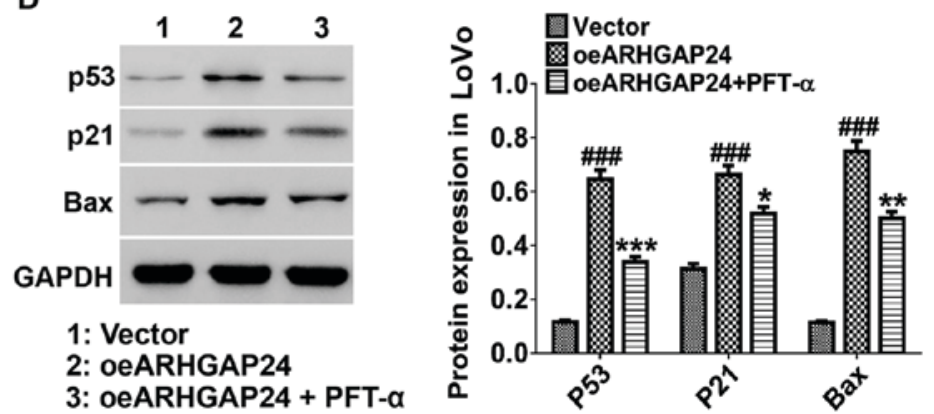

Figure 3. Upregulation of ARHGAP24 inhibits the cell ability of colorectal cancer cells via p53, p21 and Bax expression LoVo and HCT116 cells were infected with lentivirus for $0,24,48$, and $72 \mathrm{~h}$ before treated with CCK-8. (A and B) The cell ability of ARHGAP24-infected LoVo and HCT116 cells was evaluated by a microplate reader at $450 \mathrm{~nm}$. (C) ARHGAP24-infected LoVo cells were treated with p53 inhibitor PFT- $\alpha$ and then the cell ability was assessed. (D) The protein levels of $\mathrm{p} 53, \mathrm{p} 21$ and Bax were detected. Data are mean \pm standard deviation. ${ }^{*} \mathrm{P}<0.05,{ }^{* *} \mathrm{P}<0.01,{ }^{* * *} \mathrm{P}<0.001,{ }^{* * * *} \mathrm{P}<0.0001$ compared to control; ${ }^{\# \#} \mathrm{P}<0.01$,

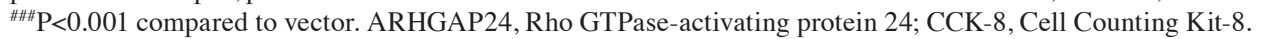
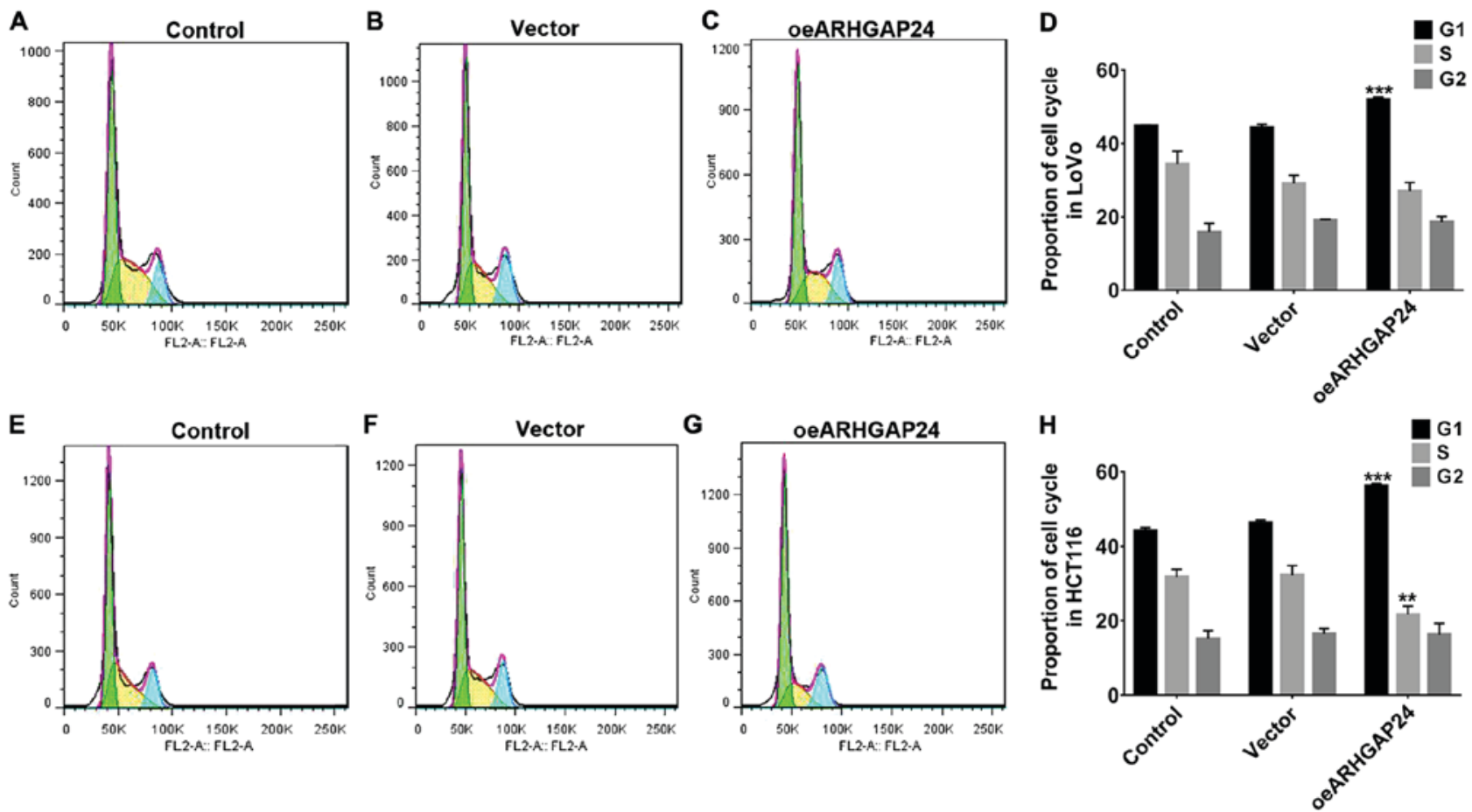

Figure 4. Upregulation of ARHGAP24 inhibited cell cycle arrest of colorectal cancer cells. In vitro, colorectal cancer cells were infected with oeARHGAP24 lentivirus for 48 h. (A-D) The cell cycle of ARHGAP24-infected LoVo cells was detected. (E-H) The cell cycle of HCT116 was also detected. All data are shown as mean \pm standard deviation. ${ }^{* *} \mathrm{P}<0.01,{ }^{* * *} \mathrm{P}<0.001$ compared to vector. ARHGAP24, Rho GTPase-activating protein 24.

the effect of oeARHGAP24 on the cell ability of LoVo cells (Fig. 3C). Simultaneously, ARHGAP24-induced the expression of p53, p21 and Bax expression was significantly decreased by PFT- $\alpha$ (Fig. 3D). These results demonstrated that ARHGAP24 regulated colorectal cancer cell proliferation probably through modulating p53, p21 and Bax expression. 

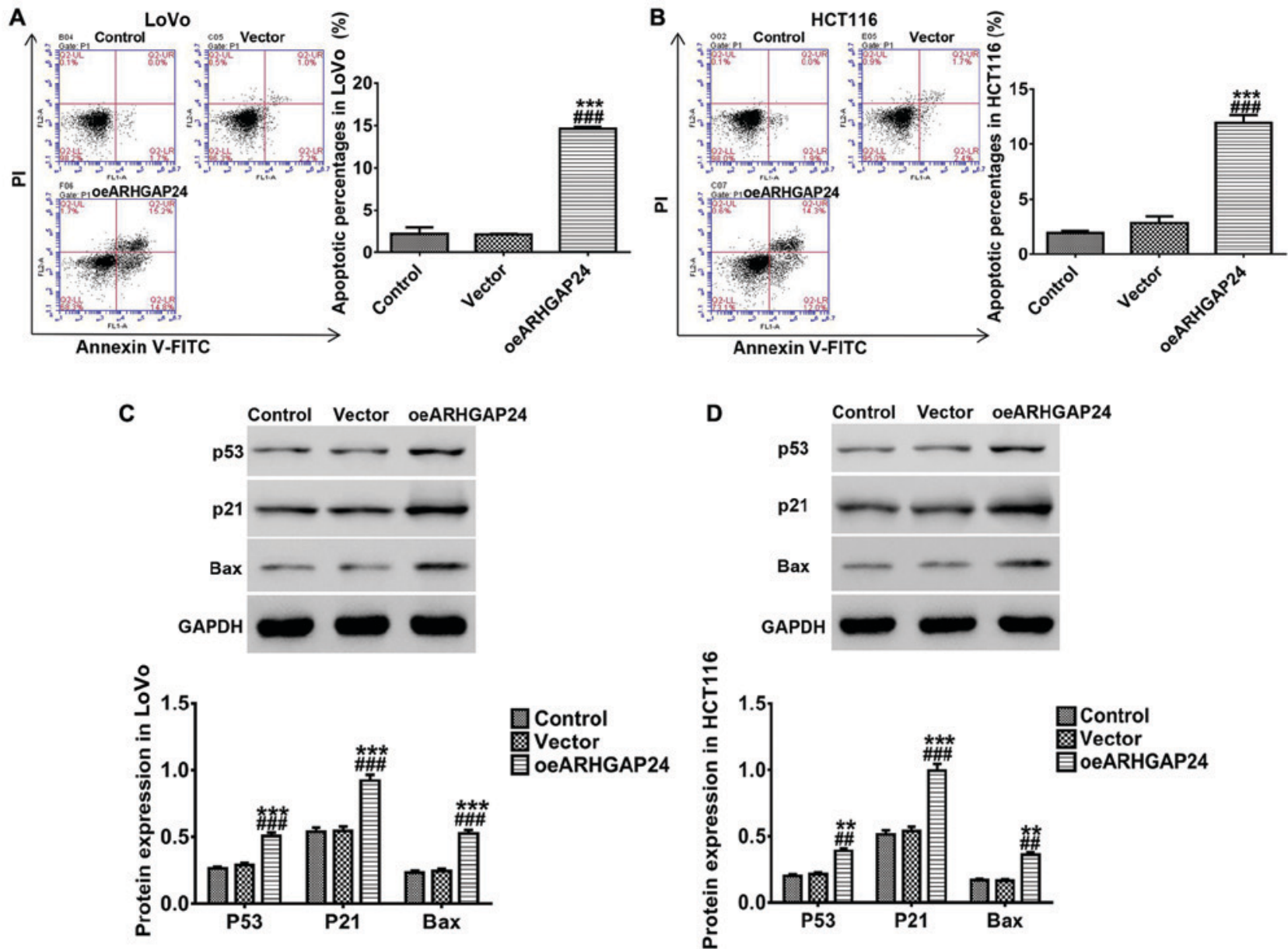

Figure 5. Upregulation of ARHGAP24 promotes apoptosis of colorectal cancer cells in vitro. LoVo and HCT116 cells were infected with lentivirus before incubated with Annexin V-FITC/PI. (A and B) After incubation, FCM analysis was employed to detect apoptotic cells. Lower right quadrant shows early apoptotic cells stained Annexin-V; upper right quadrant presents late apoptotic or necrotic cells stained with the two dyes while vital cells located in the lower left. (C and D) In addition, the expression of p53, p21 and Bax proteins were detected by western blot analysis. Data are expressed as mean \pm standard deviation. ${ }^{* *} \mathrm{P}<0.01,{ }^{* * * *} \mathrm{P}<0.001$ compared to control; ${ }^{\# \#} \mathrm{P}<0.01,{ }^{\# \#} \mathrm{P}<0.001$ compared to vector. ARHGAP24, Rho GTPase-activating protein $24 ;$ FCM, flow cytometry.

Upregulation of ARHGAP24 inhibited cell cycle arrest of colorectal cancer cells. For further investigation, after infected with oeARHGAP24, the cell cycle arrest of LoVo and HCT116 cells were detected. As shown in Fig. 5, upregulation of ARHGAP24 in LoVo cells significantly arrested the cell cycle at G1 phase, therefore reducing the proportion of cells in the S/G2 phase (Fig. 4A-D). Likewise, in HCT116 cells, ARHGAP24 upregulation showed a similar effect on cell cycle (Fig. 4E-H). These further evidenced the inhibitory effect of ARHGAP24 upregulation on the cell proliferation of colorectal cancer.

Upregulation of ARHGAP24 promotes apoptosis of colorectal cancer cells in vitro. In addition, we also studied the effect of ARHGAP24 on apoptosis of colorectal cancer cells. After infected with oeARHGAP24 lentivirus, LoVo and HCT116 cells were respectively incubated with Annex V-FITC/PI double dyes. After that, apoptotic cells were analyzed by flow cytometry. A remarkable increase of apoptosis rates was noted in LoVo cells after ARHGAP24 overexpression (Fig. 5A), and the protein levels of several apoptosis-associated proteins $\mathrm{p} 53$, p21 and Bax were increased with the increase of ARHGAP24 expression (Fig. 5C). Moreover, changes similar to those in LoVo cells also occurred in HCT116 cells (Fig. 5B and D). These indicated that upregulation of ARHGAP24 enhanced apoptosis of colorectal cancer cells which was likely to be associated with the expression of p53, p21 and Bax.

\section{Discussion}

Colorectal cancer is a human malignant tumor with high incidence and its incidence is on the rise. Due to high relapse and metastasis rate, chemical resistance and other characteristics, colorectal cancer is a serious threat to human health. Thus, more novel promising targets for colorectal cancer are urgently required to further investigate its pathogenesis. In the present study, we found that in colorectal cancer patients, the expression of ARHGAP24 and p53 in tumors was much lower than that in normal tissues, and in vitro, overexpression of ARHGAP24 remarkably inhibited the cell ability of colorectal cancer cells, arrested the cell cycle at G1 phase, reducing the proportion of cells in S/G2 phase, and accelerated the cell apoptosis probably through modulating p53, p21 and Bax expression. 
Up to date, several members of RHOGAP proteins were reported to be involved in colorectal cancer such as ARHGAP35, and ARHGAP8. A previous study revealed that the methylation in the promoter region of ARHGAP28 may affect in metastatic ability of colorectal cancer (20). In our study, declined ARHGAP24 and p53 in colorectal cancer tumor may be associated with the progress of colorectal cancer which is likely to be related to p53. Further in vitro, ARHGAP24 upregulation inhibited the cell ability of colorectal cancer cells and arrested the cell cycle at G1 phase, whereas apoptotic cells were increased, concurrent with an increase in p53, p21 and Bax expression, which suggested that ARHGAP24 may be used as a tumor suppressor in colorectal cancer through the regulation of p53, p21 and Bax. It is known that apoptosis and growth arrest are essential to the process of various cancers that occur in human. Proteins p53, p21 and Bax were more heavily acting in key roles in growth arrest and apoptosis. p53 often plays an essential role in tumor via the induction of apoptosis (21). Similar to p53, another apoptosis-related protein, Bax, belonged to $\mathrm{Bcl} 2$ family, is known as the main effecter of apoptosis and the activity of Bax is enhanced in p53-induced tumor cell apoptosis (22-24), thus, Bax is activated by p53 to take part in multiple processes such as apoptotic program $(25,26)$. In addition, tumor growth suppressor p21, downstream of p53, is also an effector gene activated by $\mathrm{p} 53$, which is implicated in cell cycle and may participate in induction of p53-dependent apoptosis. It is revealed that $\mathrm{p} 21$ can bind cyclin-dependent kinases to repress the phosphorylation of cell cycle-required proteins such as pRb, which may be induced by p53-dependent apoptosis (27-29). That is to say, through regulation of p21 and Bax, p53 performed the most important role in the regulation of the cell growth arrest and cell apoptosis in the progress of cancers (30-32). It is consistent with our results that the addition of p53 inhibitor PFT- $\alpha$ showed an antagonistic effect on oeARHGAP24-induced cell ability of colorectal cancer. The expression of p21 and Bax reduced by PFT- $\alpha$ further demonstrated that p53 had an activation effect on the expression of p21 and Bax, which indicated that the function of p53 in the cell proliferation and apoptosis closely related to $\mathrm{p} 21$ and Bax.

In conclusion, this study demonstrated that overexpression of ARHGAP24 may suppress the survival of colorectal cancer cells by regulating the cell ability and apoptosis via the modulation of p53, p21 and Bax. Therefore, ARHGAP24 may be considered as a novel promising target for the further research of colorectal cancer.

\section{Acknowledgements}

Not applicable.

\section{Funding}

The study was supported by grants from Key disciplines Group Construction Project of Pudong Health Burea of Shanghai (PWZxq2014-12), Natural Science Foundation of China (no. 81571718), Budgetary fund of Shanghai University of Traditional Chinese Medicine (2016YSN67) and Talents Training Program of Seventh People's Hospital of Shanghai University of TCM (grant no. QMX2017-01).

\section{Availability of data and materials}

The datasets used and/or analyzed during the present study are available from the corresponding author on reasonable request.

\section{Authors' contributions}

SZ and LS were responsible for cell culture and construction of lentivirus. JZ helped with immunohistochemical detection. SH performed PCR. YS and YY contributed to CCK-8 assay and western blot analysis. WX was in charge of Flow Cytometry analysis. All authors read and approved the final study.

\section{Ethics approval and consent to participate}

The study was approved by the Ethics Committee of The Seventh People's Hospital of Shanghai University of Traditional Chinese Medicine (Shanghai, China) and informed consents were signed by the patients or the guardians.

\section{Patient consent for publication}

Not applicable.

\section{Competing interests}

The authors declare that they have no competing interests.

\section{References}

1. Jemal A, Center MM, DeSantis C and Ward EM: Global patterns of cancer incidence and mortality rates and trends. Cancer Epidemiol Biomarkers Prev 19: 1893-1907, 2010.

2. Favoriti P, Carbone G, Greco M, Pirozzi F, Pirozzi RE and Corcione F: Worldwide burden of colorectal cancer: A review. Updates Surg 68: 7-11, 2016.

3. Marley AR and Nan H: Epidemiology of colorectal cancer. Int J Mol Epidemiol Genet 7: 105-114, 2016.

4. Gaedcke J, Grade M, Camps J, Søkilde R, Kaczkowski B, Schetter AJ, Difilippantonio MJ, Harris CC, Ghadimi BM, Møller S, et al: The rectal cancer microRNAome - microRNA expression in rectal cancer and matched normal mucosa. Clin Cancer Res 18: 4919-4930, 2012.

5. Siegel R, Ward E, Brawley O and Jemal A: Cancer statistics, 2011: The impact of eliminating socioeconomic and racial disparities on premature cancer deaths. CA Cancer J Clin 61: 212-236, 2011.

6. Merika E, Saif MW, Katz A, Syrigos K and Morse M: Review. Colon cancer vaccines: An update. In Vivo 24: 607-628, 2010.

7. Cunningham D, Atkin W, Lenz HJ, Lynch HT, Minsky B, Nordlinger B and Starling N: Colorectal cancer. Lancet 375: 1030-1047, 2010

8. Xi ZW, Xin SY, Zhou LQ, Yuan HX, Wang Q and Chen KX: Downregulation of rho-associated protein kinase 1 by miR-124 in colorectal cancer. World J Gastroenterol 21: 5454-5464, 2015.

9. Nakamura Y, Nishi M, Fukuda Y, Ogino M and Kosuga H: Case of the multiple liver metastases from colon cancer obtained long-term disease-free survival with multimodality therapy. Gan To Kagaku Ryoho 39: 2228-2230, 2012 (In Japanese).

10. Luo N, Guo J, Chen L, Yang W, Qu X and Cheng Z: ARHGAP10, downregulated in ovarian cancer, suppresses tumorigenicity of ovarian cancer cells. Cell Death Dis 7: e2157, 2016.

11. Teng JP, Yang ZY, Zhu YM, Ni D, Zhu ZJ and Li XQ: The roles of ARHGAP10 in the proliferation, migration and invasion of lung cancer cells. Oncol Lett 14: 4613-4618, 2017.

12. Choi EJ, Kim MS, Song SY, Yoo NJ and Lee SH: Low frequent mutation of ARHGAP35, a candidate tumor suppressor gene, in gastric and colorectal cancers. Pathol Oncol Res 24: 175-176, 2018. 
13. Johnstone CN, Castellví-Bel S, Chang LM, Bessa X, Nakagawa H, Harada H, Sung RK, Piqué JM, Castells A and Rustgi AK: ARHGAP8 is a novel member of the RHOGAP family related to ARHGAP1/CDC42GAP/p50RHOGAP: Mutation and expression analyses in colorectal and breast cancers. Gene 336: 59-71, 2004.

14. Xu G, Lu X, Huang T and Fan J: ARHGAP24 inhibits cell cycle progression, induces apoptosis and suppresses invasion in renal cell carcinoma. Oncotarget 7: 51829-51839, 2016.

15. Muller PAJ, Vousden KH and Norman JC: p53 and its mutants in tumor cell migration and invasion. J Cell Biol 192: 209-218, 2011.

16. Dang WQ, Tang H, Cao H, Wang L and Chen TM: Construction of lentivirus-mediated short hairpin RNA targeting human STAT3 gene. Xi Bao Yu Fen Zi Mian Yi Xue Za Zhi 28: 1204-1207, 2012 (In Chinese).

17. Livak KJ and Schmittgen TD: Analysis of relative gene expression data using real-time quantitative PCR and the 2(- $\Delta \Delta C(T))$ method. Methods 25: 402-408, 2001.

18. Tan Z, Liu X, Yu E, Wang H, Tang L, Wang $\mathrm{H}$ and Fu C: Lentivirus-mediated RNA interference of tripartite motif 68 inhibits the proliferation of colorectal cancer cell lines SW1116 and HCT116 in vitro. Oncol Lett 13: 2649-2655, 2017.

19. Zhu K, Guo J, Wang H and Yu W: FRAT1 expression regulates proliferation in colon cancer cells. Oncol Lett 12: 4761-4766, 2016.

20. Kasuya K, Nagakawa Y, Hosokawa Y, Sahara Y, Takishita C, Nakajima T, Hijikata Y, Soya R, Katsumata K and Tsuchida A: RhoA activity increases due to hypermethylation of ARHGAP28 in a highly liver-metastatic colon cancer cell line. Biomed Rep 4 335-339, 2016.

21. Kastan MB, Canman CE and Leonard CJ: P53, cell cycle control and apoptosis: Implications for cancer. Cancer Metastasis Rev 14: 3-15, 1995.

22. Vaseva AV and Moll UM: The mitochondrial p53 pathway. Biochim Biophys Acta 1787: 414-420, 2009.

23. Czabotar PE, Lessene G, Strasser A and Adams JM: Control of apoptosis by the BCL-2 protein family: Implications for physiology and therapy. Nat Rev Mol Cell Biol 15: 49-63, 2014.
24. Kim EM, Jung CH, Kim J, Hwang SG, Park JK and Um HD: The p53/p21 complex regulates cancer cell invasion and apoptosis by targeting Bcl-2 family proteins. Cancer Res 77: 3092-3100, 2017.

25. Chipuk JE, Kuwana T, Bouchier-Hayes L, Droin NM, Newmeyer DD, Schuler M and Green DR: Direct activation of Bax by p53 mediates mitochondrial membrane permeabilization and apoptosis. Science 303: 1010-1014, 2004.

26. Zhao G, Zhu Y, Eno CO, Liu Y, Deleeuw L, Burlison JA, Chaires JB, Trent JO and Li C: Activation of the proapoptotic $\mathrm{Bcl}-2$ protein $\mathrm{Bax}$ by a small molecule induces tumor cell apoptosis. Mol Cell Biol 34: 1198-1207, 2014.

27. Bukholm IK and Nesland JM: Protein expression of p53, p21 (WAF1/CIP1), bcl-2, Bax, cyclin D1 and pRb in human colon carcinomas. Virchows Arch 436: 224-228, 2000.

28. Katsumata K, Sumi T, Tomioka H, Aoki T and Koyanagi Y: Induction of apoptosis by p53, bax, bcl-2, and p21 expressed in colorectal cancer. Int J Clin Oncol 8: 352-356, 2003.

29. el-Deiry WS, Harper JW, O'Connor PM, Velculescu VE, Canman CE, Jackman J, Pietenpol JA, Burrell M, Hill DE, Wang Y, et al: WAF1/CIP1 is induced in p53-mediated G1 arrest and apoptosis. Cancer Res 54: 1169-1174, 1994.

30. Kanavaros P, Stefanaki K, Valassiadou K, Vlachonikolis J, Mavromanolakis M, Vlychou M, Kakolyris S, Gorgoulis V, Tzardi M and Georgoulias V: Expression of p53, p21/waf, bcl-2, bax, $\mathrm{Rb}$ and $\mathrm{Ki} 67$ proteins in colorectal adenocarcinomas. Med Oncol 16: 23-30, 1999.

31. Miyashita T, Krajewski S, Krajewska M, Wang HG, Lin HK, Liebermann DA, Hoffman B and Reed JC: Tumor suppressor p53 is a regulator of bcl-2 and bax gene expression in vitro and in vivo. Oncogene 9: 1799-1805, 1994.

32. Miyashita T and Reed JC: Tumor suppressor p53 is a direct transcriptional activator of the human bax gene. Cell 80: 293-299, 1995. 